EPJ Web of Conferences 114, 02063 (2016)

DOI: $10.1051 /$ epjconf/201611402063

C Owned by the authors, published by EDP Sciences, 2016

\title{
Optimization of ejector design and operation
}

\author{
Konstantin Kuzmenko ${ }^{1, a}$, Nina Yurchenko ${ }^{1}$, Pavlo Vynogradskyy ${ }^{1}$, Yuriy Paramonov ${ }^{1}$ \\ ${ }^{1}$ Laboratory for Advanced Aerodynamics, Institute of Hydromechanics, Nat. Academy of Sci., 8/4 Zheliabov St., Kiev 03680, Ukraine
}

\begin{abstract}
The investigation aims at optimization of gas ejector operation. The goal consists in the improvement of the inflator design so that to enable 50 liters of gas inflation within $\sim 30$ milliseconds. For that, an experimental facility was developed and fabricated together with the measurement system to study pressure patterns in the inflator path.
\end{abstract}

\section{Background}

Supersonic gas ejectors are known to be applied in various fields of industry. Their operation is based on energy transfer from pressurized gas to a high-speed flow at lower pressure $[1,2]$. The process entrains not only the gas from the high-pressure source but also the surrounding gas at normal pressure, thereby increasing a total amount (mass/volume) of gas which is to be pumped into a certain container. An aspiration coefficient is the main criterion of the system efficiency; it is defined by a ratio of the total amount of pumped gas to the amount of pressurized gas.

\section{Experimental facility}

An experiment facility was designed and fabricated for testing developed ejector system in a process of their characteristics improvement (Fig. 1). It consists of the following basic parts: high-pressure tank (250 bar), receiver for averaged and high pressure (150 bar), pressure gauges, two high-speed valves, data acquisition and processing system, small-size ejector for flowrate testing, high- and low-pressure compressors, etc. To model the ejection process needed air consumption with cold air two high-speed valves were used. The air is pumped into the $40 \mathrm{~L}$ high-pressure tank by high-pressure PE-100W compressor up to 100 bar. From this tank, air goes to the $40 \mathrm{~L}$ receiver through a reduction valve. The reduction valve is adjusted to the range of pressure values required in experiments. Pressure in the receiver is measured using two pressure gauges: the first one up to 40 bars, the second one up to 100 bars. Both gauges indications do not differ more than 0.5 bar during the experiment. The drainage crane is provided for air exhaust in case of arising overpressure in the receiver. From the receiver, air can flow in one of two pipes leading to a particular high-speed valve. The first KB-20 high-speed valve has an opening time of $2 \mathrm{~ms}$ in a pressure range of 1-70 bars. The second ARMA highspeed valve was found to have an opening time of 4-6 ms in the pressure range of 1-36 bars. Specified highpressure sensors for 1-70 bars are mounted after each valve to measure pressure losses.

\subsection{High-speed valves}

To satisfy the task requirements of $50 \mathrm{~L}$ inflation within $30 \mathrm{~ms}$, high-speed valve had to be used as a heart of the inflation system. Two high-speed valves were used for

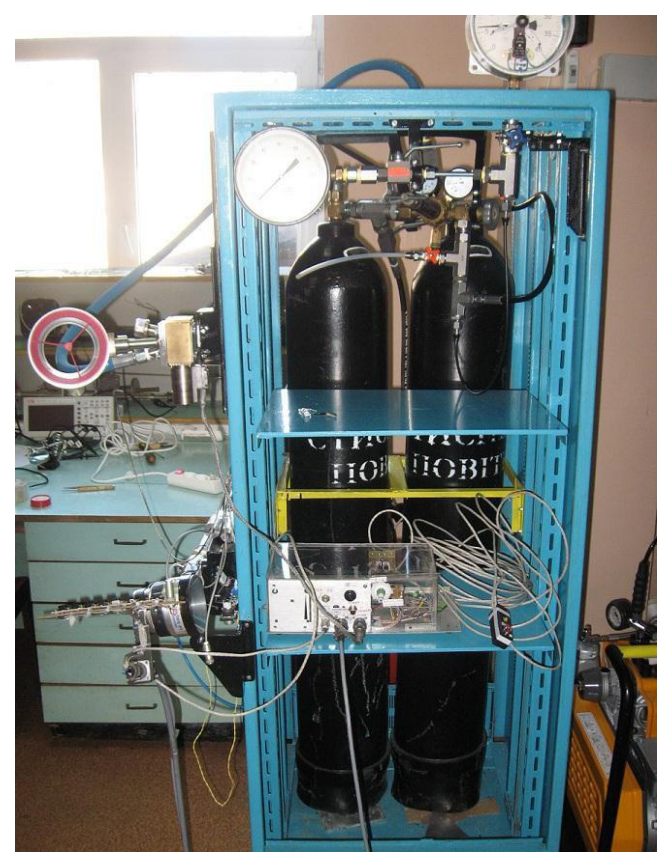

Figure 1. Experimental facility

ejection process investigation with compressed air. First one KB-20 (ISTA Inc., St. Petersburg, Russia, http://www.ista-pneumatics.ru/ru/kv.shtml).

\footnotetext{
$\bar{a}$ Corresponding author: k_kuzmenko@ex.ua
} 


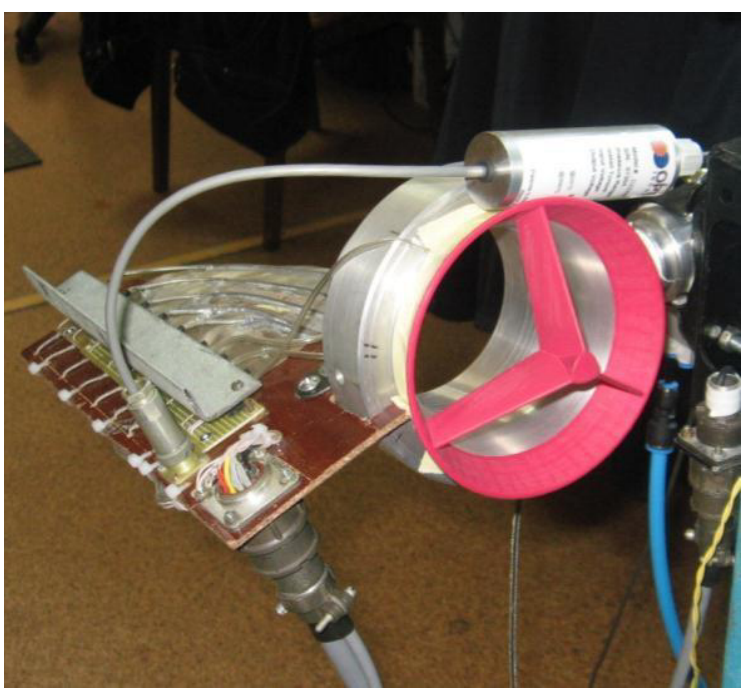

Figure 2. Inlet of the ejector.

The second valve is designed at the specialized ARMA enterprise (Central design bureau of valves, Kiev, Ukraine). Unlike the first valve, The ARMA valve makes its further development possible with parameters adjusted to the future tasks. At present, the key parameters are as follows.

Valve opening time is 4-6 ms;

system response time (between an initiating electric signal and the valve opening) is $0.01 \div 0.012 \mathrm{~s}$;

throat diameter is $12 \mathrm{~mm}$;

operating pressure range is $0.1 \div 3.5 \mathrm{MPa}$.

This valve has an electrical control unit which provides both manual and remote control of its operation within 20 to $50 \mathrm{~ms}$.

A basic difference of the two valves design is their throat diameter of $20 \mathrm{~mm}$ (KB-20) and $12 \mathrm{~mm}$ (ARMA valve). It has an essential impact on a flow rate in the inflator system that is important to seek for ways of the inflation system improvement. That is why both kinds of the highspeed valves were integrated into the facility and thoroughly studied. Valve opening time can be varied in a wide range of 20-50 ms. Present experiments were carried out at $30 \mathrm{~ms}$. It was chosen from a viewpoint of the formulated problem, of technical characteristics of available high-speed valves as well as of prospective investigations to follow.

\subsection{Measurement system}

The available PCI-1747U 64-channel analog input board with up to $250 \mathrm{kS} / \mathrm{s}$ sampling rate provides sufficient measuring capabilities along with Advantech AIMB782QG2-00A1E motherboard and Intel Core i7-4790K processor. Such configuration of the data acquisition and processing system enables measurements of up to 25 parameters with time resolution of $0.1 \mathrm{~ms}$. It is considered quite sufficient providing up to 1000

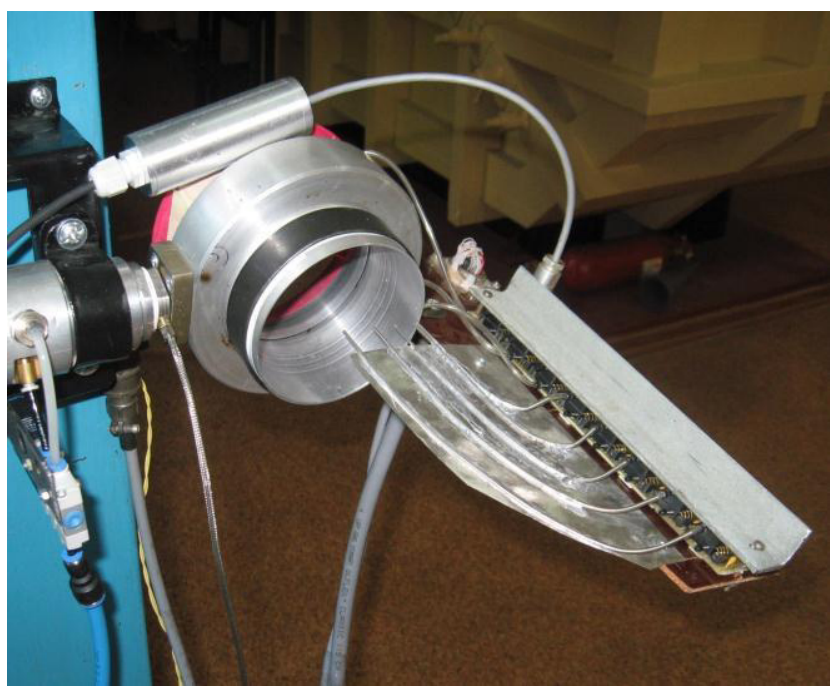

Figure 3. Pressure measuring comb.

measurements of each parameter during the ejection process cycle.

For the flowrate measurements, 6 sensors are mounted at the ejector outlet. The designed pressure sensor comb combines 6 sensor tubes. Three of them are located near a wall; one is situated on an ejector outlet axis. OPTrand sensor (pressure range $0 \div 70$ bar) is placed between the valve outlet and the ejector inlet. Three sensors are flushmounted in the ejector internal tract for static pressure measurement. For velocity field measurements, five more pressure probes (Fig. 3, 5) are mounted at the side to avoid their influence on exhaust flow from the ejector outlet. Full pressure probes are mounted on a holder under various steps. Analog and digital signals from sensors go to the centralized Data Acquisition and Processing Complex.

Fig. 4 shows typical data obtained from a highpressure

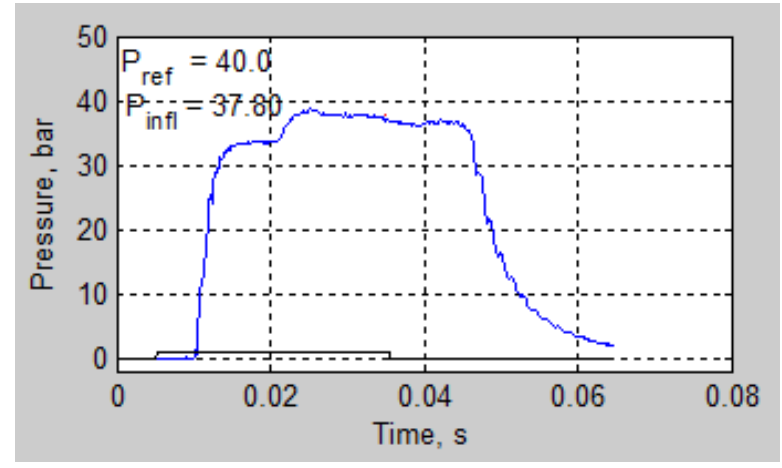

Figure 4. Pressure after valve (valve opening moment)

sensor. It demonstrates the high-speed valve operation and time when the pressure reaches its maximum.

Fig. 2 shows the ejector experimental model. Its inlet diameter is $13 \mathrm{~mm}$, outlet diameter is $84 \mathrm{~mm}$. Slit width can be changed within $0.095-0.25 \mathrm{~mm}$. According to technical requirements, the overall ejector size (diameter) must be less than $150 \mathrm{~mm}$, and the length should not exceed $120 \mathrm{~mm}$. 


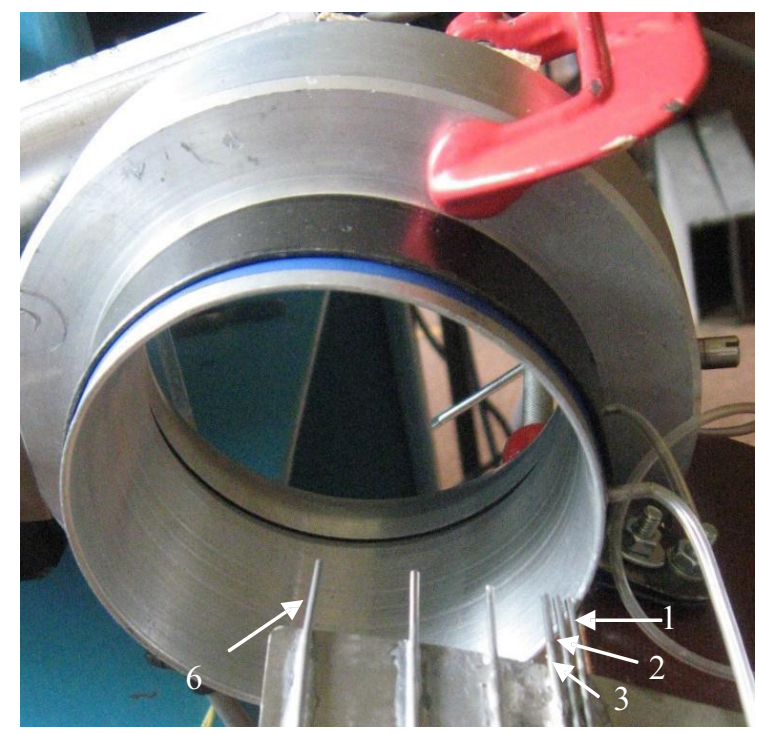

Figure 5. Pressure comb. Position \#1-3 is near wall pressure probes, probe \#6 is on the axis of ejector outlet

\section{Results}

Tests were performed at different pressure ranges and cycle durations. A basic phenomenon which was studied under testing was jet separation. The separation restricts the arbitrary pressure growth aimed to increase higher flow rates of ejected air. Thus the work is focused on jet

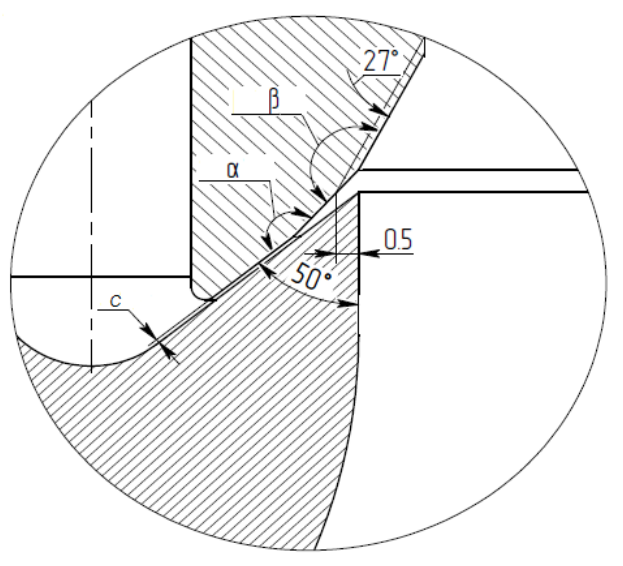

Figure 6. Initial slit dimensions

separation delay with increased pressure, because if it is so there is no ambient air sucked in ejector.

\subsection{First experimental series}

Experimental investigation started with a slit width of $0.25 \mathrm{~mm}$ decreasing to $0.12 \mathrm{~mm}$ in this test series. At this configuration, separation takes place at low pressure values (14.5 and 17.8 bar respectively). After that, the slit width was decreased to $0.08 \mathrm{~mm}$. The latter resulted in jet separation started at 25 bar and ended up at 29 bar. It was called as 'Full separation' characterizing the situation of complete jet separation from the wall. The 'Full separation' velocity is registered with a sensor located at the ejector outlet axis. To ensure accuracy and repeatability of measurement results, the pressure sensor \#3 was connected to the third near-wall tube. Fig. 6 shows the experimental ejector arrangement.

\subsection{Jet separation determination}

Jet separation was detected by pressure drop at near wall sensors and simultaneously pressure increasing at sensors located at the ejectors outlet axis. For example separation occurrence at small pressure increasing (from 40 to 40.5 bars) is shown at Fig. 8. and 9. Simultaneously with this process sensors located near at ejector axis detect air jet. This is shown at Fig. 10 and Fig. 11. Static pressure measurement sensor located at ejector inlet also detect jet separation this results are shown in Fig. 12-14.

\subsection{Second experimental series}

The second cycle of measurements was carried out with the changed slit configuration. Two slit angles were changed to values of $\alpha=20^{\circ}, \beta=23^{\circ}$. The sharp edge of the slit projecting part has been reduced to 0.5 $\mathrm{mm}$, so sharp edge get lower than bending point of $\beta$ angle. Experiment test was performed for three slit width $0.1 \mathrm{~mm}, 0.14$ and $0.25 \mathrm{~mm}$. At slit width $0.1 \mathrm{~mm}$ and new slit shape contour the jet separation appears at pressure values more than 30 bars (at pressure equal to 30.36 bar

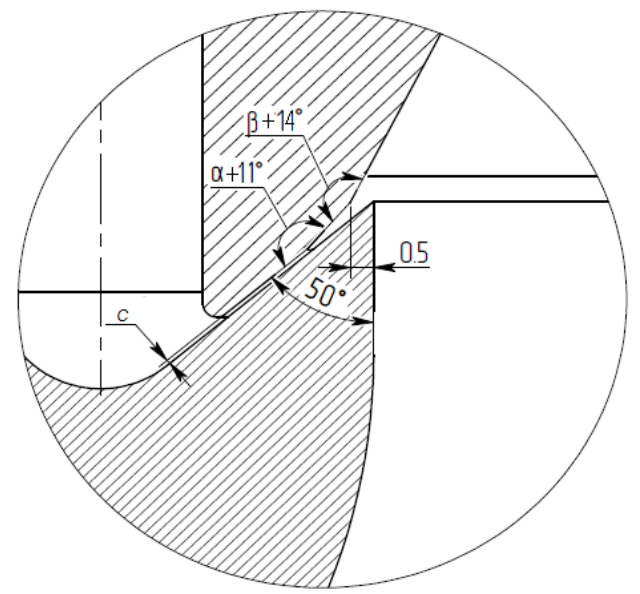

Figure 7. Second version of ejector slit

near-wall jet is already attached to wall) also vacuum pressure at the ejector inlet (sensor \#1) reaches 0.08 bar. But small pressure increasing to 30.57 bar leads to separation phenomena and full separation happens at 31 32 bar. Slit dimension after first changing is shown in Fig. 7.

\subsection{Third experimental series}

Second ejector version was made based on previous results. Main changes were: increased angles: $\alpha$ to $20^{\circ}, \beta$ to $5^{\circ}$. Experiment series were made at slit width $0.1 \mathrm{~mm}$ and $0.21 \mathrm{~mm}$. Under this geometry configuration (at slit width $0.1 \mathrm{~mm}$ ) the separation occurs in range from 40 to 


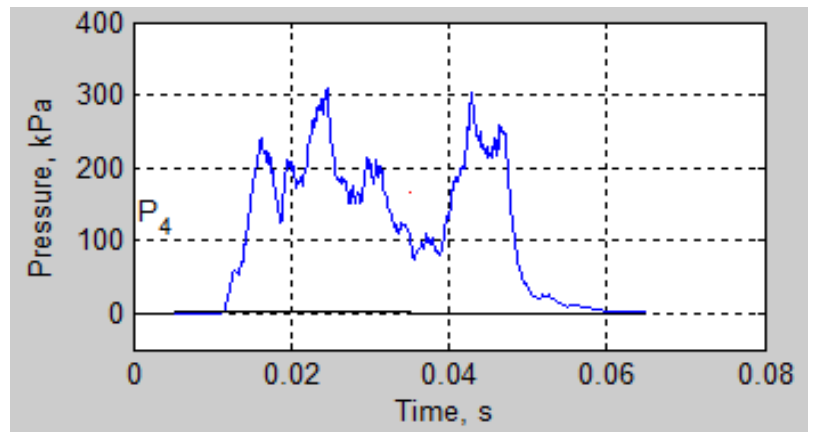

Figure 8. Near-wall sensor data at pressure 40 bar

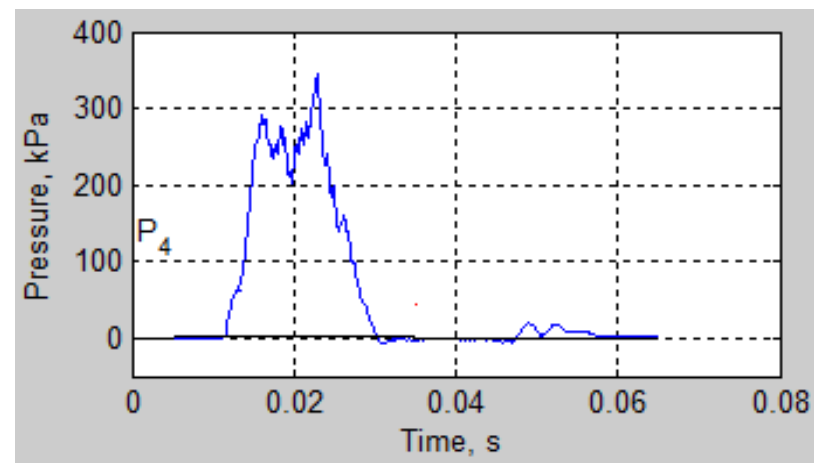

Figure 9. Near-wall sensor data at pressure 40.5 bar

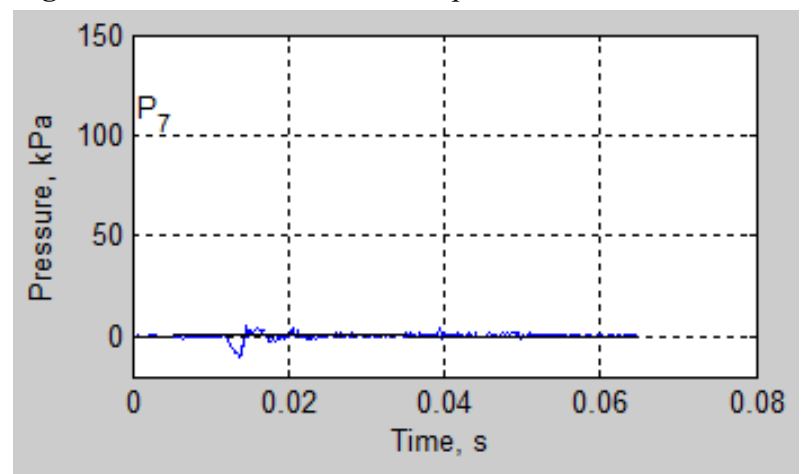

Figure 10. Axis sensor data at pressure $40 \mathrm{bar}$

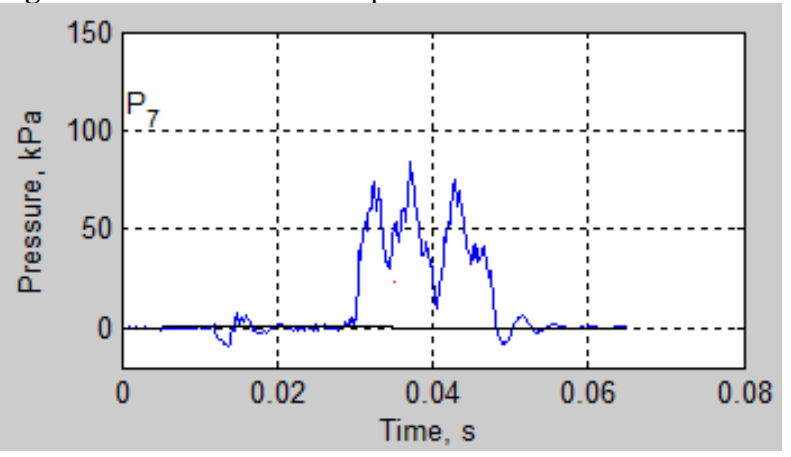

Figure 11. Axis sensor data at pressure 40.5 bar

40.5 bars. Experiment was made also with slit width $0.21 \mathrm{~mm}$ and showed high vacuum pressure value at the ejector inlet. Sucked jet velocity in ejector reached 160 $\mathrm{m} / \mathrm{s}$ at the vacuum pressure about 0.17 bar.

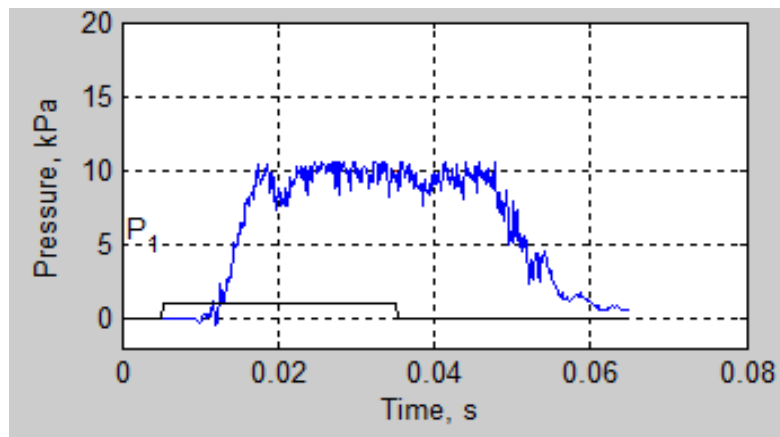

Figure 12. Pressure at the ejector inlet, $P=30$ bar

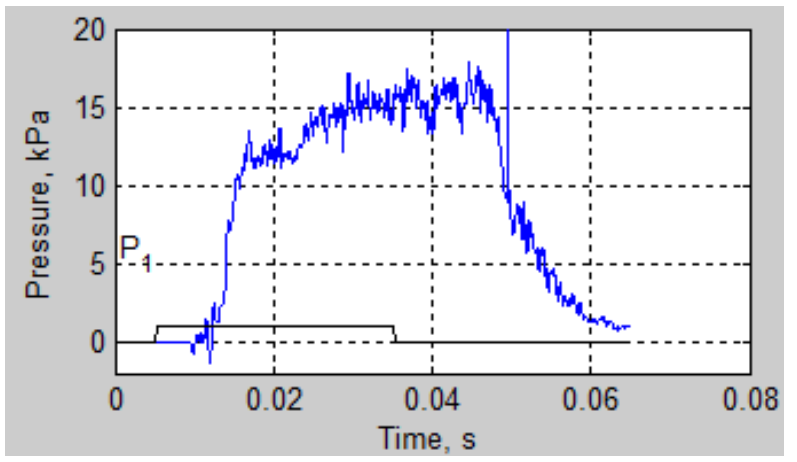

Figure 13. Pressure at the ejector inlet, $\mathrm{P}=39 \mathrm{bar}$

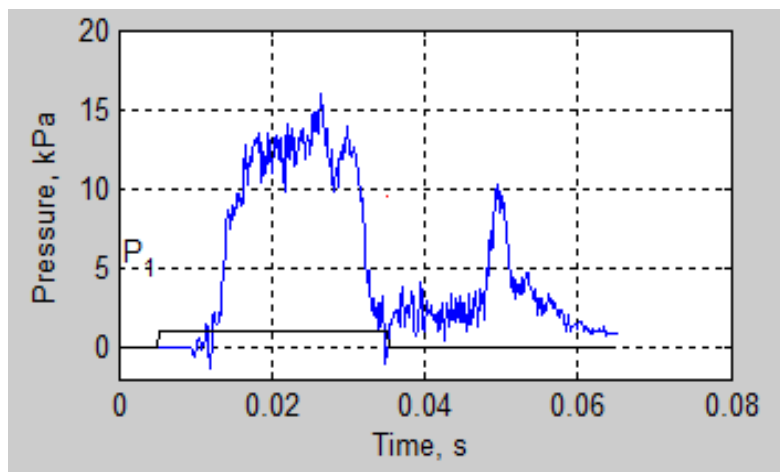

Figure 14. Pressure at the ejector inlet, $P=40.5$ bar

Conclusions. To optimize gas ejector operation, the experimental facility was designed together with the remote data acquisition and processing system. The ejector test model was designed under guidance of numerical results modeling the ejection process. Three experimental test series resulted in the successive ejector geometry optimization. As a result, jet separation occurred at $\sim 3$ times higher pressure ( 40.5 bar) compared to the initial case (17 bar). The work is in progress.

\section{References}

1. E.Y.Sokolov, H.M.Zinger, Jet devices (Government energetic publishing, Moscow, 1960) In Russian

2. Y.K.Arkadov, New gas ejector (Phys.-Math lit., Moscow, 2001) In Russian. 